CARDIOVASCULAR MEDICINE

\title{
Predictors of poor mid-term health related quality of life after primary isolated coronary artery bypass grafting surgery
}

\author{
S Al-Ruzzeh, T Athanasiou, O Mangoush, J Wray, T Modine, S George, M Amrani
}

Heart 2005;91:1557-1562. doi: 10.1136/hrt.2004.047068

See end of article for authors' affiliations

.....................

Correspondence to: Mr Sharif Al-Ruzzeh, Leeds General Infirmary, 18 Fielding Way, Leeds LS27 9AB, UK:

sharifalruzzeh@

hotmail.com

Accepted 24 January 2005

\begin{abstract}
Objective: To assess the determinants of poor mid-term health related quality of life (HRQoL) at one year after primary isolated coronary artery bypass grafting (CABG).

Methods: 463 patients who underwent primary isolated CABG for multivessel disease and came for their annual follow up at the outpatient clinic during one year at Harefield Hospital, Middlesex, were approached to participate in the present study. Prospective clinical data were collected as part of the clinical care of the patients and were retrospectively analysed when the patients consented to participate in the study at their outpatient visit. After their consent they were given three HRQoL assessment questionnaires. Scores, together with clinical data, were analysed by both univariate and multivariate analyses with regard to poor HRQoL outcome.

Results: 437 (94.4\%) patients consented to participate in the study and filled in the HRQoL questionnaires. Ten variables were identified in the univariate analysis as potential predictors of poor scores of the physical element of HRQoL; however, only three variables-gastrointestinal problems, congestive heart failure, and type D personality trait-predicted poor physical scores independently. Eleven variables were identified in the univariate analysis as potential predictors of poor scores of the mental element of HRQoL; however, only three variables-peripheral vascular disease, infective complications, and type D personality trait-predicted poor physical scores independently.

Conclusion: Preoperative gastrointestinal problems, preoperative congestive heart failure, and type $D$ personality trait were independent predictors of the poor physical component of HRQoL. Peripheral vascular disease, infective complications, and type $D$ personality trait were independent predictors of the poor mental component of HRQoL. Interestingly, patients with type $D$ personality were more than twice as likely to have poor physical HRQoL and more than five times as likely to have poor mental HRQoL.
\end{abstract}

$\mathrm{T}$ he World Health Organization defines quality of life as "an individual's perception of their position in life in the context of the culture and value systems in which they live and in relation to their goals, expectations, standards and concerns"; in other words, it is a person's perception of his or her being. The concept of health related quality of life (HRQoL) was derived from that general definition and has been increasingly attracting consideration in the provision of health care. It gains its importance in coronary artery bypass grafting (CABG) surgery from the fact that the goal of such surgery is to improve quality of life and restore general well being, mainly through alleviation of symptoms, particularly angina, which is achievable in up to $80 \%$ of the patients. ${ }^{1}$ The simple outcome measurements of morbidity and mortality are insufficient in assessing the real and full picture of the effect of CABG or any other procedure or intervention on patients, particularly in the presence of huge pressures on health care providers to run their services under certain economic constrains but still observe high level quality assurance. ${ }^{2}$ On the other hand, HRQoL assessment is rather complicated by its subjective nature. ${ }^{3}$

The goal of the present study was to assess the determinants of poor mid-term HRQoL at one year after primary isolated CABG.

\section{PATIENTS AND METHODS \\ Data collection}

Four hundred and sixty three patients, who underwent primary isolated $C A B G$ for multivessel disease and came for their annual follow up at the outpatient clinic during one year at Harefield Hospital, Middlesex, were approached to participate in the present study. All the clinical data were collected prospectively in line with the appended minimum dataset defined by the Society of Cardiothoracic Surgeons of Great Britain and Ireland. The collected data are regularly validated locally and validated on a $3-5$ yearly cycle by the Society. Those prospectively collected clinical data were retrieved from registry database, medical notes, outpatient notes, and intensive therapy unit (ITU) charts. The data were retrospectively analysed when the patients consented to participate in the study at their outpatient visit. Institutional approval was obtained for the study.

\section{HRQoL assessment}

Assessment of HRQoL was planned in a cross sectional survey format. All eligible patients were approached and given a brief explanation of the study and, if they consented to participate, they were given three self administered questionnaires to fill in: the 36 item short form health survey

\footnotetext{
Abbreviations: $C A B G$, coronary artery bypass grafting; $C H F$, congestive heart failure; DS14, type D scale-14; GIT, gastrointestinal tract; HADS, hospital anxiety and depression scale; HRQoL, health related quality of life; IHD, ischaemic heart disease; ITU, intensive therapy unit; MSC, mental component summary; NA, negative affectivity; NYHA, New York Heart Association; PCS, physical component summary; PVD, peripheral vascular disease; SF-12, 12 item short form health survey; SF-36, 36 item short form health survey; SI, social inhibition
} 
(SF-36), the hospital anxiety and depression scale (HADS), and Denollet's personality type D scale-14 (DS14).

The SF-36 is a validated questionnaire widely used in medical practice and research, which has been used with cardiac surgery patients. ${ }^{45}$ It consists of 36 items measuring eight domains of HRQoL: physical functioning, social functioning, role limitations due to physical problems, role limitations due to emotional problems, mental health, energy and vitality, body pain, and general health. Two summary scores are calculated from the eight domains' scores. The physical component summary (PCS) score summarises four domains' scores: physical functioning, role limitations due to physical problems, body pain, and general health. The mental component summary (MCS) score summarises the other four domains' scores: social functioning, role limitations due to emotional problems, mental health, and energy and vitality. The two summary scores were calculated according to the method described previously by Ware et al. ${ }^{6}$ In the absence of recognised standards of high and low HRQoL scores, we dichotomised the PCS and MCS scores into high and low after assuming an elective cut off point based on the scores of the whole study group. The lower $33 \%$ of the PCS and MCS scores were regarded as low, which is a method that has been previously used by others to categorise the HRQoL performance. ${ }^{4}$ The wording of six items on the SF-36 were modified slightly to make it acceptable for British patients.

The HADS questionnaire is a 14 item scale originally developed to assess both anxiety and depression in an outpatient setting irrespective of any physical symptoms. ${ }^{8}$ It consists of seven items to measure anxiety and seven items to measure depression. Each item is scored from 0 to 3, with total scores ranging from 0 to 21 for the anxiety and depression subscales. Higher scores indicate greater anxiety and depression. The four score ranges are classified as normal $(0-7)$, mild (8-10), moderate (11-14), and severe (15-21). To simplify the statistical analysis, we grouped patients into those with scores from $0-10$ as normal and those with scores from 11-21 as being significantly anxious or depressed.

The DS14 comprises 14 questions divided into two subscales: negative affectivity (NA) and social inhibition

Table 1 Univariate analysis of all variables for high and low scores in the physical (PCS) and mental component summary (MCS) scores of the 36 item short form health survey (SF-36)

\begin{tabular}{|c|c|c|c|c|c|c|c|}
\hline \multirow[b]{2}{*}{ Variable } & \multirow[b]{2}{*}{ Total $(n=437)$} & \multicolumn{3}{|l|}{ PCS } & \multicolumn{3}{|l|}{ MCS } \\
\hline & & High $(n=279)$ & Low $(n=158)$ & p Value & High $(n=286)$ & Low $(n=151)$ & p Value \\
\hline Female sex & 79 (18.1\%) & 48 & 31 & 0.53 & 50 & 29 & 0.66 \\
\hline Age at surgery $>70$ & $137(31.3 \%)$ & 88 & 49 & 0.91 & 100 & 37 & 0.03 \\
\hline Symptom status (stable) & $202(46.2 \%)$ & 127 & 75 & 0.7 & 122 & 80 & 0.04 \\
\hline Diabetes & $87(19.9 \%)$ & 47 & 40 & 0.03 & 57 & 30 & 0.99 \\
\hline Hypertension & $228(52.2 \%)$ & 139 & 89 & 0.19 & 142 & 86 & 0.16 \\
\hline Hypercholesterolaemia & $270(61.8 \%)$ & 173 & 97 & 0.9 & 176 & 94 & 0.88 \\
\hline Smoking & $274(62.7 \%)$ & 174 & 100 & 0.85 & 170 & 104 & 0.05 \\
\hline Preoperative AF & $11(2.5 \%)$ & 7 & 4 & 1 & 8 & 3 & 0.75 \\
\hline Postoperative AF & $99(22.7 \%)$ & 63 & 36 & 0.96 & 67 & 32 & 0.6 \\
\hline Renal problems & $2(0.5 \%)$ & 1 & 1 & 1 & 2 & 0 & 0.54 \\
\hline GIT problems & $88(20.1 \%)$ & 48 & 40 & 0.04 & 61 & 27 & 0.39 \\
\hline Respiratory problems & $25(5.7 \%)$ & 12 & 13 & 0.09 & 11 & 14 & 0.03 \\
\hline PVD & $32(7.3 \%)$ & 15 & 17 & 0.04 & 16 & 16 & 0.05 \\
\hline Preoperative MI & $157(35.9 \%)$ & 96 & 61 & 0.4 & 101 & 56 & 0.71 \\
\hline Last $\mathrm{MI}>30$ days & $191(43.7 \%)$ & 119 & 72 & 0.56 & 126 & 65 & 0.84 \\
\hline Perioperative $\mathrm{MI}$ & $4(0.9 \%)$ & 4 & 0 & 0.3 & 4 & 0 & 0.3 \\
\hline Thrombolysis within last 24 hours & $15(3.4 \%)$ & 9 & 6 & 0.75 & 8 & 7 & 0.32 \\
\hline Previous failed PTCA/stent & $49(11.2 \%)$ & 29 & 20 & 0.47 & 33 & 16 & 0.77 \\
\hline Poor LV function (EF <30\%) & $22(5 \%)$ & 12 & 10 & 0.35 & 13 & 9 & 0.52 \\
\hline Angina (CCS III-IV) & $206(47.1 \%)$ & 126 & 80 & 0.27 & 128 & 78 & 0.17 \\
\hline Dyspnoea (NYHA III/IV) & $85(19.5 \%)$ & 45 & 40 & 0.02 & 50 & 35 & 0.15 \\
\hline Congestive heart failure & $43(9.8 \%)$ & 21 & 22 & 0.03 & 22 & 21 & 0.04 \\
\hline IV nitrates & $38(8.6 \%)$ & 20 & 18 & 0.13 & 20 & 18 & 0.08 \\
\hline Cardiogenic shock (IV inotropes) & $3(0.7 \%)$ & 3 & 0 & 0.56 & 3 & 0 & 0.56 \\
\hline Preoperative IABP & $14(3.2 \%)$ & 10 & 4 & 0.78 & 9 & 5 & 1 \\
\hline Postoperative IABP & $17(3.9 \%)$ & 11 & 6 & 0.94 & 13 & 4 & 0.33 \\
\hline Preoperative CVA or TIA & $18(4.1 \%)$ & 7 & 11 & 0.02 & 8 & 10 & 0.05 \\
\hline Postoperative CVA or TIA & $5(1.1 \%)$ & 2 & 3 & 0.36 & 4 & 1 & 0.66 \\
\hline Elective priority & $116(26.5 \%)$ & 73 & 43 & 0.81 & 76 & 40 & 0.99 \\
\hline $\mathrm{BSA}<2 \mathrm{~m}^{2}$ & $261(59.7 \%)$ & 168 & 93 & 0.78 & 176 & 85 & 0.29 \\
\hline $\mathrm{BMI}>30 \mathrm{~kg} / \mathrm{m}^{2}$ & $128(29.3 \%)$ & 73 & 55 & 0.05 & 79 & 49 & 0.29 \\
\hline Use of radial artery graft & $210(48.1 \%)$ & 140 & 70 & 0.27 & 151 & 77 & 0.72 \\
\hline Cardiopulmonary bypass & $201(46 \%)$ & 131 & 70 & 0.59 & 137 & 64 & 0.27 \\
\hline Left main stem disease & $41(9.4 \%)$ & 25 & 16 & 0.69 & 23 & 18 & 0.19 \\
\hline Ventilation $>24$ hours & $44(10.1 \%)$ & 32 & 12 & 0.2 & 32 & 12 & 0.28 \\
\hline Low cardiac output & $27(6.2 \%)$ & 18 & 9 & 0.75 & 19 & 8 & 0.58 \\
\hline Infective complications & $25(5.7 \%)$ & 11 & 14 & 0.03 & 11 & 14 & 0.02 \\
\hline Pulmonary complications & $36(8.2 \%)$ & 21 & 15 & 0.47 & 17 & 19 & 0.02 \\
\hline Renal complications & $31(7.1 \%)$ & 20 & 11 & 0.94 & 23 & 8 & 0.29 \\
\hline GIT complications & $11(2.5 \%)$ & 8 & 3 & 0.75 & 8 & 3 & 0.76 \\
\hline Sternal resuturing & $12(2.7 \%)$ & 5 & 7 & 0.11 & 6 & 6 & 0.25 \\
\hline Readmission to ITU & $6(1.4 \%)$ & 1 & 5 & 0.03 & 5 & 1 & 0.67 \\
\hline Reoperation for bleeding & $26(6 \%)$ & 17 & 9 & 0.86 & 16 & 10 & 0.67 \\
\hline Length of stay $>7$ days & $422(96.6 \%)$ & 266 & 156 & 0.06 & 274 & 148 & 0.23 \\
\hline Type D personality & 150 (34.3\%) & 77 & 73 & $<0.001$ & 59 & 91 & $<0.001$ \\
\hline Anxiety & 429 (98.2\%) & 275 & 154 & 0.47 & 284 & 145 & 0.02 \\
\hline Depression & $435(99.5 \%)$ & 277 & 158 & 0.54 & 286 & 149 & 0.12 \\
\hline
\end{tabular}




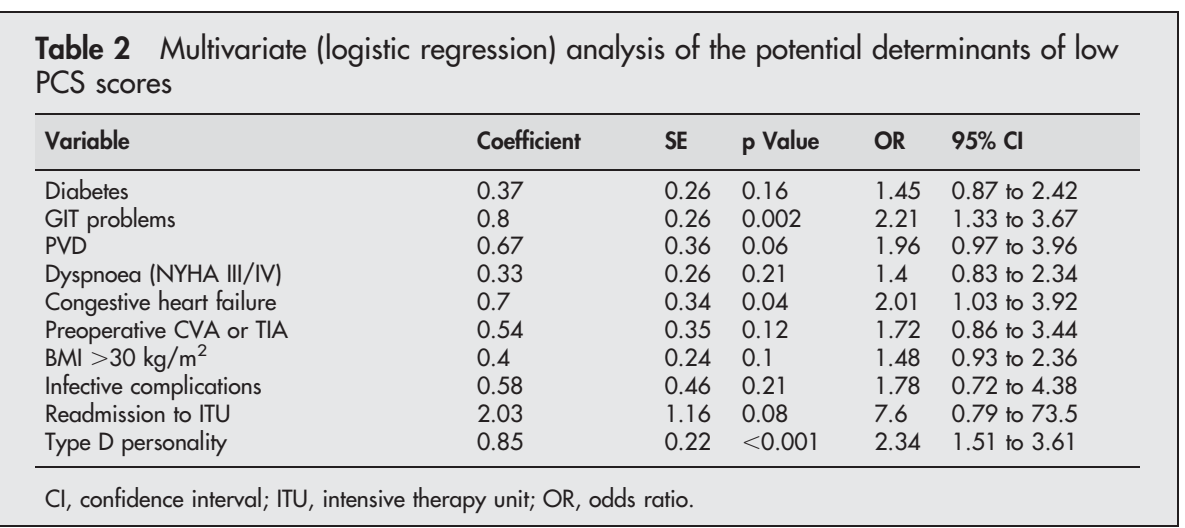

(SI). Each has seven questions. The answer to each question is scored from 0 to 4 with total scores ranging from 0 to 28 for the NA and SI subscales. Patients are classified as having "type D personality" if both NA and SI scores are 10 or greater. The NA questions measure the tendency to experience negative emotions across times and situations and the SI questions measure the tendency to inhibit the expression of emotions and behaviours in social interactions. ${ }^{9}$

\section{Statistical analysis}

Patient preoperative characteristics, operative variables, and postoperative complications were compared by univariate analysis, with Fisher's exact test or $\chi^{2}$ test where appropriate, with regard to high and low PCS and MCS scores of the SF36 questionnaire. Values of $p \leqslant 0.05$ indicated a significant difference. Variables that proved to be significant $(p \leqslant 0.05)$ were considered potential predictors of low PCS and MCS scores and were included in the multivariate logistic regression analysis to determine the independent predictors of poor HRQoL in this group of patients.

Participants greatly outnumbered non-participants. To facilitate comparison between the two groups a nonparametric bootstrap technique was used by generating a large number of datasets by sampling with replacement from the original sample of non-participants. This method works by constructing lots of datasets (1000 in the present study) similar to the actual dataset and then using these datasets to summarise the parameters of interest. Data were initially preprocessed on Microsoft Excel 2000 (Microsoft Corp, Redmond, Washington, USA) and subsequently exported into SPSS version 11 for Windows (SPSS Inc, Chicago, Illinois, USA) and Intercooled Stata 6.0 for Windows (StataCorp LP, College Statins, Texas, USA). Bootstrap simulations were done with Resampling Stats in Excel version 2 (Resampling Stats Inc, Arlington, Virginia, USA).

\section{RESULTS}

Four hundred and thirty seven (94.4\%) patients consented to participate in the study and filled in the HRQoL questionnaires. Using the bootstrap method for comparison between non-participants and participants we did not identify any significant differences in sociodemographic parameters (age, sex, marital status, education $>10$ years, employment status-for example, not working and retired from work) and clinical parameters (severity of angina status, extent of coronary disease, number of grafts, and postoperative length of stay).

The mean (SD) age at surgery was 65.24 (8.51) years (range 38-84 years). They had a mean body surface area of $1.92(0.2) \mathrm{m}^{2}$ (range $1.21-2.5 \mathrm{~m}^{2}$ ) and a mean body mass index of 27 (4) $\mathrm{kg} / \mathrm{m}^{2}$ (range $16.9-51.12 \mathrm{~kg} / \mathrm{m}^{2}$ ). The 201 $(46 \%)$ patients who had cardiopulmonary bypass during surgery had a cumulative bypass time of 83.83 (36.38) minutes and a cumulative aortic cross clamp time of 28.1 (21.38) minutes. Table 1 shows other patient characteristics including preoperative and operative variables and postoperative complications. Appendix 1 lists definitions of the variables.

The univariate analysis showed that patients with low PCS scores differed significantly from patients with high PCS scores on 10 variables, which were considered potential predictors of low PCS scores. These were diabetes, gastrointestinal tract (GIT) problems, peripheral vascular disease (PVD), dyspnoea with New York Heart Association (NYHA) functional classes III and IV, congestive heart failure (CHF), preoperative cerebrovascular accidents or transient ischaemic attacks, body mass index $>30 \mathrm{~kg} / \mathrm{m}^{2}$, infective complications, readmission to the ITU, and type D personality trait. Only three variables-GIT problems, CHF, and type D personality trait-predicted low PCS scores independently (table 2). Eighty eight patients had preoperative GIT

\begin{tabular}{|c|c|c|c|c|c|}
\hline Variable & Coefficient & SE & $p$ Value & OR & $95 \% \mathrm{Cl}$ \\
\hline Age $>70$ & -0.46 & 0.25 & 0.07 & 0.63 & 0.39 to 1.04 \\
\hline Dyspnoea (NYHA III/IV) & 0.09 & 0.28 & 0.74 & 1.1 & 0.63 to 1.91 \\
\hline Smoking & 0.15 & 0.13 & 0.22 & 1.17 & 0.91 to 1.49 \\
\hline Respiratory problems & 0.69 & 0.47 & 0.14 & 1.99 & 0.79 to 4.99 \\
\hline PVD & 0.91 & 0.41 & 0.03 & 2.49 & 1.11 to 5.57 \\
\hline Congestive heart failure & 0.56 & 0.37 & 0.14 & 1.74 & 0.84 to 3.62 \\
\hline Preoperative CVA or TIA & 0.48 & 0.59 & 0.42 & 1.61 & 0.5 to 5.1 \\
\hline Infective complications & 0.67 & 0.25 & 0.007 & 1.96 & 1.2 to 3.19 \\
\hline Pulmonary complications & 0.69 & 0.41 & 0.1 & 1.99 & 0.89 to 4.45 \\
\hline Type D personality & 1.7 & 0.24 & $<0.001$ & 5.51 & 3.49 to 8.73 \\
\hline Anxiety & -1.6 & 0.92 & 0.08 & 0.2 & 0.03 to 1.22 \\
\hline
\end{tabular}


problems: 15 patients had hiatus hernia, three of whom had previous corrective surgery; 62 patients had diagnosed peptic ulcer disease; and 11 patients had GIT bleeding with normal endoscopic results and therefore no definite diagnosis was made.

The univariate analysis showed that patients with low MCS scores differed significantly from patients with high MCS scores in 11 variables, which were considered potential predictors of low MCS scores. These were age $>70$, dyspnoea (NYHA III/IV), smoking, respiratory problems, PVD, CHF, preoperative cerebrovascular accidents or transient ischaemic attacks, infective complications, pulmonary complications, type D personality trait, and anxiety. Only three variablesPVD, infective complications, and type D personality traitpredicted low MCS scores independently (table 3 ). Infective complications occurred in 25 patients: 12 patients developed deep sternal wound infections that required resuturing; three patients developed septicaemia; and 10 patients developed leg vein harvest wound infection.

\section{DISCUSSION}

This cross sectional survey study, planned in a descriptive correlational design, aimed at assessing the determinants of poor mid-term HRQoL at one year after primary isolated CABG. The study showed that preoperative GIT problems, CHF, and type D personality trait were independent predictors of the poor physical component of HRQOL. PVD, infective complications, and type D personality trait were independent predictors of the poor mental component of HRQoL. One interesting finding of the present study was that patients with type D personality were more than twice as likely to have poor physical HRQoL and more than five times as likely to have poor mental HRQoL than patients without type D personality, independent of all other preoperative, operative, and postoperative variables.

Most patients with cardiac disease undergo CABG for prognostic reasons irrespective of the level of their cardiac symptoms. Randomised controlled studies in the 1970s and early ' 80 s provided the most reliable data that support the survival benefit of CABG surgery versus medical treatment. ${ }^{10}$ This survival benefit seemed to be more apparent in moderate risk and high risk patients than in low risk patients. ${ }^{11}$ However, the survival benefit was not the only favourable outcome of CABG surgery over medical treatment: relief of symptoms and improved quality of life were also obtained. ${ }^{12}$

In the present study, GIT problems predicted a poor physical component of HRQoL. In patients with ischaemic heart disease (IHD) diagnosed either by exercise test or angiography, most chest and epigastric pains are primarily attributed to their "more serious" IHD rather than to other known or unknown GIT problems. This may delay or mask the diagnosis, and consequently treatment, of the relatively simple GIT problem and thus the quality of life of these patients may decrease further. On the other hand, the unstable course of IHD and its accompanying psychological and physical stresses may be responsible for the relapsing behaviour of the peptic ulcer disease. ${ }^{13}$ Thus, such an association between the two may be synergistic, but it remains difficult to ascertain which of the two is responsible for the bigger portion of the symptom burden, particularly pain. ${ }^{14}$ We believe that among the patients in our study who reported a poor physical component of their HRQoL after CABG, GIT problems were responsible for a major portion of their symptom burden to start with.

CHF, in the present study patients, was diagnosed clinically in the preoperative period, either by the referring cardiologist or by the operating surgeon, and the patient was labelled to have CHF irrespective of left ventricular systolic function as assessed by preoperative angiographic examination. In a recent cross sectional study of patients with CHF being considered for heart transplantation, the degree of CHF was correlated with the PCS and MCS scores of SF-36. Those MCS scores were relatively more affected, ${ }^{15}$ in contrast to our study, where CHF predicted only low PCS scores. Furthermore, the degree of CHF in that study was defined by several clinical indicators, among which was NYHA class III or IV. In our study, NYHA class III or IV on its own was significant in the univariate analysis but was not an independent predictor of poor HRQoL. More in-depth research into the HRQoL of this particular group of patients showed that some sort of cross link and integration exists between the mental and physical components of this type of patients' quality of life. ${ }^{16}$ These patients can get more depressed as a result of their physical symptoms and this in turn causes a sharp decline in both their physical and mental HRQoL. ${ }^{17}$ Evidence still justifies operating on patients with advanced ischaemic CHF on the basis that even if their left ventricular function does not improve they still experience less risk of infarction and death. ${ }^{18}$

Postoperatively, it is frustrating for patients with IHD and PVD that they are restricted not by their cardiac function anymore but by ischaemic leg pains due to intermittent claudication. It is not surprising that a recent comparative study found that the impact of PVD on almost all the SF-36 domains of HRQoL was more significant than that of the IHD itself. ${ }^{19}$ Therefore, it is not surprising that, in the present study, patients with documented PVD preoperatively reported about two and a half times worse mental HRQoL than did patients without PVD. Those patients were found to have, in addition to the physical limitation, frustration, pain, limitation in social functioning, uncertainty, and fear, all of which contributed to the psychosocial and emotional impairment. ${ }^{20}$

Infective complications had a significant impact on the mental component of HRQoL. A recent study showed that surgical site infections had a major effect on the HRQoL, assessed by the 12 item short form health survey (SF-12), in a group of orthopaedic surgery patients and that was correlated with length of stay, readmission, and cost. ${ }^{21}$ Furthermore, surgical site wound infections, diagnosed after discharge, still had the same negative effect on self reported HRQoL, as shown by a recent big community based study of several subgroups of surgical patients after hospital discharge, including a subgroup of CABG patients. ${ }^{22}$

A significant finding of the present study is that patients with type $\mathrm{D}$ personality were more than twice as likely to have poor physical HRQoL and more than five times as likely to have poor mental HRQoL than patients without type D personality trait. In fact, type D personality trait was the only common predictor for both poor physical and poor mental HRQoL outcome.

Type D personality, where the letter D refers to "distressed", is a stable personality profile characterised by both the tendency to experience negative emotions (NA subscale) and the tendency to inhibit self expression in social interaction (SI subscale). ${ }^{9}$ Such people experience high levels of chronic tension and anger, depressive symptoms, poor self esteem, dissatisfaction with life in general, low positive affect, and lack of social support..$^{22}$ This personality trait has been correlated with fatal and non-fatal cardiac events, poor HRQoL, and inadequate response to treatment in several types of patients with IHD, including patients after an MI and those with poor left ventricular function. ${ }^{23}{ }^{24}$ However, to our knowledge, this is the first study to show that type D personality is also correlated with poor physical and mental HRQoL after CABG surgery. Recent evidence suggested a psychosomatic relation between type D personality and several active neuroendocrine and immune mechanisms found in these patients that may explain such findings. ${ }^{25} 26$ 
The prevalence of relatively high anxiety and depression scores, as measured by HADS, among our study patient group conforms to similar previous reports in the literature. These two factors did not show up in this study as significant predictors of poor HRQoL. ${ }^{27}$ However, poor HRQoL is different from the psychiatric clinical diagnosis of depression, which was found to be strongly correlated with HRQoL and clinical outcomes after CABG surgery. ${ }^{28}$

In conclusion, this cross sectional survey study showed that patients with type D personality are more likely to have poor physical and mental components of HRQoL. However, further prospective studies are required to shed more light on the effect of the type D personality trait on HRQoL after CABG surgery. This will help to identify the group of patients at risk of developing suboptimal HRQoL postoperatively, who can then be targeted by various programmes of psychotherapy and behavioural therapy preoperatively, and perhaps to realistically predict HRQoL outcome after CABG surgery.

One important limitation of this study is its cross sectional design. Thus, by definition the study did not measure HRQoL preoperatively (baseline), which usually is the major determinant of postoperative HRQoL. ${ }^{29}$ Therefore, we believe that a longitudinal sectional study comparing preoperative with postoperative HRQoL and its relation to other variables would be more valuable in this respect. However, the study still has some value in helping realise the factors that can affect the quality of life of this particular group of patients in the midterm.
Authors' affiliations

S Al-Ruzzeh, Leeds General Infirmary, Leeds, UK

T Athanasiou, O Mangoush, J Wray, T Modine, S George, M Amrani,

The National Heart and Lung Institute, Imperial College of Science,

Technology and Medicine, Harefield Hospital, Middlesex, UK

\section{REFERENCES}

1 Klonoff H, Clark C, Kavanagh-Gary D, et al. Two-year follow-up study of coronary bypass surgery: psychologic status, employment status and quality of life. J Thorac Cardiovasc Surg 1989;97:78-85.

2 Speziale G, Bilotta F, Ruvolo G, et al. Return to work and quality of life measurement in coronary artery bypass grafting. Eur J Cardiothorac Surg $1996 ; 10: 852-8$.

3 Bowling A. What things are important in people's lives? Survey of the public's judgements to inform scales of health-related quality of life. Soc Sci Med 1995;41:1447-62.

4 Simchen E, Galai N, Braun D, et al. Sociodemographic and clinical factors associated with low quality of life one year after coronary bypass operations: the Israeli coronary artery bypass study (ISCAB). J Thorac Cardiovasc Surg 2001;121:909-19.

5 Permanyer $M$, Brotons $C$, Cascant $P$, et al. Assessment of quality of life related health 2 years after coronary surgery. Med Clin (Barc) 1997; 108:446-51.

6 Ware J, Kosinski M, Keller S. SF-36 physical and mental health summary scales: a user's manual, 2nd ed. Boston: The Health Institute, New England Medical Center, 1994.

7 Jenkinson C, Coulter A, Wright L. Short form 36 (SF 36) health survey questionnaire: normative data for adults of working age. BMJ 1993;306:1437-40.

8 Zigmond AS, Snaith RP. The hospital anxiety and depression scale. Acta Psychiatr Scand 1983;67:361-70.

9 Denollet J, Vaes J, Brutsaert D. Inadequate response to treatment in coronary heart disease: adverse effects of type $D$ personality and younger age on 5year prognosis and quality of life. Circulation 2000;102:630-5.

\section{APPENDIX}

\section{Definitions of variables}

\begin{tabular}{|c|c|}
\hline Variable & Definition \\
\hline Symptom status (stable) & No recent deterioration and no unstable angina \\
\hline Smoking & Past or present \\
\hline \multirow[t]{2}{*}{ Renal problems } & - Serum creatinine $\geqslant 200 \mu \mathrm{mol} / \mathrm{I}$ \\
\hline & - Acute or chronic renal failure requiring haemodialysis \\
\hline \multirow[t]{4}{*}{ GIT problems } & - Diagnosed hiatus hernia \\
\hline & - Diagnosed peptic ulcer disease \\
\hline & - Diagnosed pancreatitis \\
\hline & - GIT bleeding \\
\hline Respiratory problems & - Chronic obstructive airway disease \\
\hline & - Bronchial asthma requiring the use of inhalers \\
\hline Peripheral vascular disease & Chronic ischaemia of lower limbs \\
\hline \multirow[t]{4}{*}{ Perioperative MI } & Presence of at least one of the following: \\
\hline & - New $Q$ waves in the ECG \\
\hline & - $\mathrm{CK}-\mathrm{MB}>50$ with ECG changes \\
\hline & - $\mathrm{CK}-\mathrm{MB}>70$ without ECG changes \\
\hline Cardiogenic shock (iv inotropes) & Up till operating theatre \\
\hline CVA or TIA & $\begin{array}{l}\text { - TIA when the neurological deficit lasted }<24 \text { hours } \\
\text { - CVA when the neurological deficit lasted }>24 \text { hours }\end{array}$ \\
\hline Low cardiac output & $\begin{array}{l}\text { In absence of the previously described perioperative } \mathrm{Ml} \text { criteria, when the systolic arterial blood pressure was } \\
\text { persistently }<90 \mathrm{~mm} \mathrm{Hg} \text { or the mean arterial blood pressure was persistently below }<50 \mathrm{~mm} \mathrm{Hg} \text { despite } \\
\text { appropriate fluid management and requiring inotropic or vasoconstrictor treatment }\end{array}$ \\
\hline \multirow[t]{3}{*}{ Infective complications } & - Septicaemia \\
\hline & - Sternal wound infection \\
\hline & - Conduit harvest site infection \\
\hline \multirow[t]{4}{*}{ Pulmonary complications } & - Adult respiratory distress syndrome \\
\hline & - Reintubation/tracheostomy \\
\hline & - Pleural effusion \\
\hline & - Pneumothorax \\
\hline Renal complications & New occurrence of one of the following: \\
\hline & - Serum creatinine $\geqslant 200 \mu \mathrm{mol} / \mathrm{l}$ \\
\hline & - Renal failure requiring haemodialysis \\
\hline GIT complications & Postoperative occurrence of one of following: \\
\hline & - Peptic ulceration \\
\hline & - Pancreatitis \\
\hline & - Mesenteric infarction \\
\hline & - GIT bleeding \\
\hline & - Perforation \\
\hline
\end{tabular}

CK, creatine kinase; CVA, cerebrovascular accident; GIT, gastrointestinal tract; iv, intravenous; MI, myocardial infarction; TIA, transient ischaemic attack. 
10 Anon. Coronary artery surgery study (CASS): a randomized trial of coronary artery bypass surgery: survival data. Circulation 1983;68:939-50.

11 Yusuf S, Zucker D, Peduzzi P, et al. Effect of coronary artery bypass graft surgery on survival: overview of ten-year results from randomized trials by the Coronary Artery Bypass Graft Surgery Trialist Collaboration. Lancet 1994;344:563-70.

12 Myers W, Schaff H, Fisher L, et al. Time to first myocardial infarction in patients with severe angina and three-vessel disease comparing medical and early surgical therapy: a CASS registry study of survival. J Thorac Cardiovasc Surg 1988:95:382-9.

13 Osadchii V, Chernin V, Chervinets V. Features of mucosal flora associated with relapse of ulcers and acute erosive-ulcer damage to the gastroduodenal zone during an unstable course of ischemic heart disease. Eksp Klin Gastroenterol. 2002;5: 24-9, 126).

14 Baena J, Lopez D, De La Poza M, et al. Association between ischemic heart disease and peptic ulcer: a case-control study. Gastroenterol Hepatol $2001 ; 24: 421-6$.

15 Westlake C, Dracup K, Creaser J, et al. Correlates of health-related quality of life in patients with heart failure. Heart Lung 2002;31:85-93.

16 Bosworth H, Steinhauser K, Orr M, et al. Congestive heart failure patients' perceptions of quality of life: the integration of physical and psychosocial factors. Aging Ment Health 2004;8:83-91.

17 Rumsfeld J, Havranek E, Masoudi F, et al. Depressive symptoms are the strongest predictors of short-term declines in health status in patients with heart failure. J Am Coll Cardiol 2003;42:1811-7.

18 Samady H, Elefteriades J, Abbot B, et al. Failure to improve left ventricular function after coronary revascularization for ischaemic cardiomyopathy is not associated with worse outcome. Circulation 1999;100:1298-304.

19 De Graaff J, Ubbink D, Kools E, et al. The impact of peripheral and coronary artery disease on health-related quality of life. Ann Vasc Surg 2002; 16:495-500.
20 Treat-Jacobson D, Halverson SL, Ratchford A, et al. A patient-derived perspective of health-related quality of life with peripheral arterial disease. J Nurs Scholarsh 2002;34:55-60.

21 Whitehouse J, Friedman N, Kirkland K, et al. The impact of surgical-site infections following orthopedic surgery at a community hospital and a university hospital: adverse quality of life, excess length of stay, and extra cost. university hospital: adverse quality of life, excess
Infect Control Hosp Epidemiol 2002;23:183-9.

22 Perencevich E, Sands K, Cosgrove S, et al. Health and economic impact of surgical site infections diagnosed after hospital discharge. Emerg Infect Dis 2003:9:196-203.

23 Denollet J, Brutsaert D. Reducing emotional distress improves prognosis in coronary heart disease: 9-year mortality in a clinical trial of rehabilitation. Circulation 2001;104:2018-23.

24 Denollet J, Brutsaert D. Personality, disease severity and the risk of long term cardiac events in patients with a decreased ejection fraction after myocardial infarction. Circulation 1998;97:167-73.

25 Habra M, Linden W, Anderson J, et al. Type D personality is related to cardiovascular and neuroendocrine reactivity to acute stress. J Psychosom Res 2003:55:235-45.

26 Denollet J, Conraads V, Brutsaert D, et al. Cytokines and immune activation in systolic heart failure: the role of type D personality. Brain Behav Immun 2003;17:304-9.

27 Pigany-Demaria V, Lesperance F, Demaria R, et al. Depression and anxiety and outcomes of coronary artery bypass surgery. Ann Thorac Surg 2003;75:314-21.

28 Connerney I, Shapiro P, McLaughlin J, et al. Relation between depression after coronary artery bypass surgery and 12-month outcome: a prospective study. Lancet 2001;358:1766-71.

29 Rumsfeld J, MaWhinney S, McCarthy M, et al. Health-related quality of life as a predictor of mortality following coronary artery bypass graft surgery. JAMA 1999;281:1298-303.

\section{IMAGES IN CARDIOLOGY}

\section{Neovascularisation of giant left atrial myxoma visualised on angiography}

A 69 year old woman presented with a two week history of dyspnoea, chest tightness, and paroxysmal atrial fibrillation. A large mass in the left atrium (LA) was detected on transthoracic echocardiography. Transoesophageal echocardiography performed to define the mass further confirmed a non-homogenous mass measuring $6 \times 5 \mathrm{~cm}$ attached to the intra-atrial septum. This mass resulted in obliteration of the LA cavity with prolapse through the mitral valve into the left ventricle during diastole (panel A: AM, atrial myxoma; LA, left atrium; $L V$, left ventricle; $R V$, right ventricle). A mean gradient of $15 \mathrm{~mm} \mathrm{Hg}$ was measured across the mitral valve and severe pulmonary hypertension was detected. Coronary angiography was performed to exclude significant coronary artery disease before surgery. On angiography, notable neovascularisation of the tumour was seen as indicated by multiple small and tortuous vessels (panel B: RCA, right coronary artery; $<$, neovascularisation of tumour). The tumour
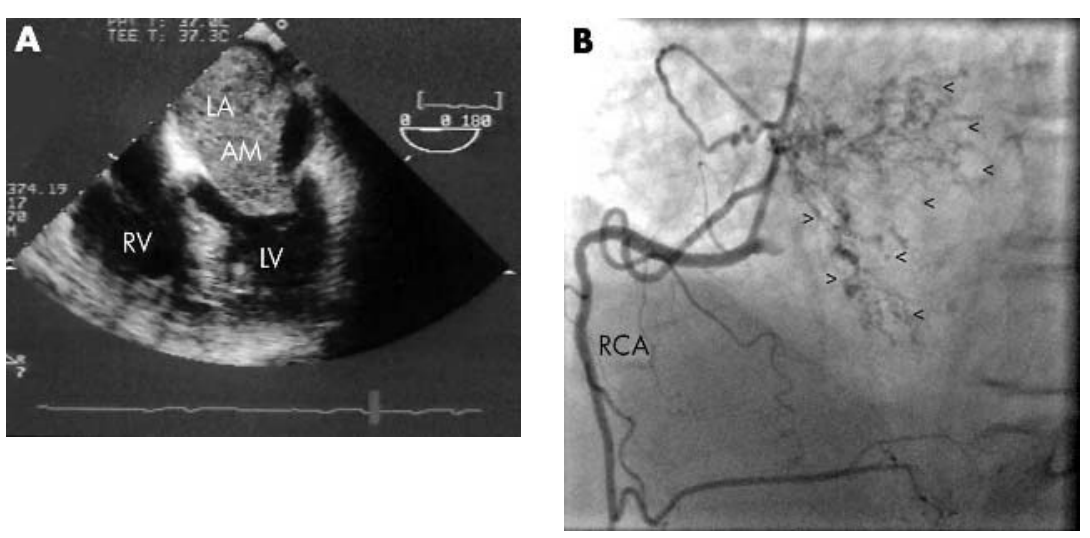

had a rich arterial supply arising predominantly from the right coronary artery and also the left circumflex artery. Surgery was performed and the tumour $(6.8 \times 5.0 \times 3.5 \mathrm{~cm})$ was successfully excised. Histology confirmed the diagnosis of cardiac myxoma. This case demonstrates that coronary angiography can provide additional information to echocardiography for the diagnosis and evaluation of atrial myxoma, by visualising arterial supply of the tumour.

B P Y Yan

A Aggarwal

R P Brown

A E Ajani andrew.ajani@mh.org.au 